\title{
Article \\ Utilizing UAVs Technology on Microscopic Traffic Naturalistic Data Acquirement
}

\author{
Apostolos Anagnostopoulos $(D)$ and Fotini Kehagia *
}

Highway Laboratory, Division of Transportation and Construction Management, School of Civil Engineering, Aristotle University of Thessaloniki, 54124 Thessaloniki, Greece; aposanag@civil.auth.gr

* Correspondence: fkehagia@civil.auth.gr; Tel.: +30-2310-994380

Citation: Anagnostopoulos, A.; Kehagia, F. Utilizing UAVs

Technology on Microscopic Traffic

Naturalistic Data Acquirement.

Infrastructures 2021, 6, 89. https:/ /

doi.org/10.3390/infrastructures6060089

Academic Editors: Krzysztof

Goniewicz, Robert Czerski and

Marek Kustra

Received: 16 May 2021

Accepted: 10 June 2021

Published: 16 June 2021

Publisher's Note: MDPI stays neutral with regard to jurisdictional claims in published maps and institutional affiliations.

Copyright: (c) 2021 by the authors. Licensee MDPI, Basel, Switzerland. This article is an open access article distributed under the terms and conditions of the Creative Commons Attribution (CC BY) license (https:// creativecommons.org/licenses/by/ $4.0 /)$.

\begin{abstract}
Research into collecting and measuring reliable, accurate, and naturalistic microscopic traffic data is a fundamental aspect in road network planning scientific literature. The vehicle trajectory is one of the main variables in traffic flow theory that allows to extract information regarding microscopic traffic flow characteristics. Several methods and techniques have been applied regarding the acquisition of vehicle trajectory. The forthcoming applications of intelligent transport systems on vehicles and infrastructure require sufficient and innovative tools to calibrate existing models on more complex situations. Unmanned aerial vehicles (UAVs) are one of the most emerging technologies being used recently in the transportation field to monitor and analyze the traffic flow. The aim of this paper is to examine the use of UAVs as a tool for microscopic traffic data collection and analysis. A comprehensive guiding framework for accurate and cost-effective naturalistic traffic surveys and analysis using UAVs is proposed and presented in detail. Field experiments of acquiring vehicle trajectories on two multilane roundabouts were carried out following the proposed framework. Results of the experiment indicate the usefulness of the UAVs technology on various traffic analysis studies. The results of this study provide a practical guide regarding vehicle trajectory acquirement using UAVs.
\end{abstract}

Keywords: UAVs; naturalistic vehicle trajectories; microscopic traffic data; traffic data collection; guiding framework

\section{Introduction}

\subsection{Microscopic Traffic Data Acquirement}

Research into collecting and measuring reliable microscopic traffic data is a fundamental aspect in road network planning scientific literature. Several applications can be implemented by using the acquired dataset in terms of traffic safety, road capacity, and level of service analysis [1-4]. However, as the traffic conditions get more complex, the level of detail and the quality of the collected information is getting higher.

The forthcoming applications of intelligent transport systems on vehicles and infrastructure mean that existing road layouts need to be examined across a wider range of scenarios [5]. As new technologies are applied to transport systems, accurate calibration and validation approaches of microscopic traffic flow models are essential. Driver behavior modelling, especially in complex scenarios and dynamic environments, such as roundabouts, is challenging and depends mainly on the size and the variety of the obtained data [6]. A dataset of accurate and high detailed microscopic traffic data can improve the reliability of models and allow a sufficient traffic analysis.

The vehicle trajectory is one of the main variables in traffic flow theory that allows to extract information regarding microscopic traffic flow characteristics [7-9]. The ability to extract the position of the vehicles over time along the roadway can provide an understanding of the way vehicles move and a comprehensive dataset for implementation in several applications regarding traffic flow and safety analysis $[10,11]$. 
Several data collection techniques are used regarding the acquisition of vehicle trajectory [12-17]. Each one is characterized by its potential capabilities and limitations. Thus, the selection of the proper technique for the execution of the survey is strongly related to the level of detail, accuracy and the purpose of the study. The most common methods, as have been identified according to the literature, are summarized in the following: (a) application of global positioning systems (GPS), (b) video processing techniques, (c) applications based on smartphone device technology, and (d) on satellite navigation systems.

However, a main challenge for researchers and traffic engineers is the ability to extract accurate and naturalistic traffic data. Data mining techniques by acquiring accurate traffic data from naturalistic driving behavior is a remarkable and active field of research during last years. Several applications can be found in the scientific literature addressing these issues $[18,19]$. Nevertheless, it is noted that in most vehicle trajectory studies, collected data lack naturalness, as experiments are conducted under the awareness of the drivers. Thus, the necessary quality of the dataset is weak in terms of representing the actual driving behavior.

In the last years, video image processing techniques are applied more frequently as they represent a low-cost and non-infrastructure-based method for acquiring naturalistic vehicle trajectories $[5,20]$. Several studies have been developed by extracting traffic data from recorded videos.

In this context, unmanned aerial vehicles (UAVs) are one of the most emerging technologies being used recently in the transportation field to monitor and analyze the traffic flow. UAV image acquisition technologies have been developed and allow to extract and analyze the traffic information in a sufficient way.

\subsection{UAV Technology for Traffic Surveys and Analysis}

UAVs can be integrated in various applications of the transport engineering sector, such as road safety inspections, traffic analysis and damage assessment for roads [21-25]. The benefits of deploying UAVs for traffic monitoring and analysis have been considered in several studies in the past few years [5,20,26-28]. As the camera is in the air, the drivers' attitude is not distracted by the equipment. According to this, extracted data represent naturalistic driving behavior which is significant for the study of vehicle trajectories. Moreover, UAVs require less experience and training to be controlled while they can handle a wide field of view, covering large areas quickly. These assets result in a timesaving, low-cost, and non-infrastructure-based technique for acquiring individual vehicle trajectories, compared to other methods.

However, as UAVs are rapidly growing in popularity and their technology is improving constantly, the implemented applications on transportation sector have not fully developed yet. Considering this, several limitations can be identified [11,29,30]. There are many factors that influence the performance of this process. Among them, weather conditions (e.g., rain), technical issues (e.g., low battery duration), and regulatory issues (e.g., no-fly zones) are the most critical to be mentioned. Thus, the ability to select and implement UAVs as an efficient traffic survey tool depends on many aspects. Table 1 summarizes the main benefits and limitations of using UAVs for traffic surveys.

Table 1. The main benefits and limitations of using UAVs for traffic surveys.

\begin{tabular}{cc}
\hline Benefits & Limitations \\
\hline UAVs require little experience & Climate characteristics \\
UAVs provide wide field of view & Technical issues of UAVs \\
UAVs can cover large areas quickly & Time constraints \\
Low cost and saving-time technique & Regulatory issues \\
Non-infrastructure-based technique & Safety issues \\
Monitoring and analysis of naturalistic & driving behavior
\end{tabular}


The aim of this paper is to examine the use of UAVs as a tool for microscopic traffic data collection and analysis. A comprehensive guiding framework for accurate and costeffective naturalistic traffic surveys using UAVs is proposed and presented in detail. An experiment involving the acquisition of vehicle trajectories on two multilane roundabouts is described. Finally, the utility of UAVs regarding microscopic traffic data acquirement is discussed based on the literature review and the analyzed guiding framework of the study.

\section{The Framework}

An extensive literature review of existing studies and frameworks regarding traffic data acquirement using UAVs was carried out $[11,15,26-28,31]$ to establish and applicate a comprehensive guiding framework in this study. A bibliometric and visualization analysis was conducted by identifying the articles with the most effort on traffic data acquirement using UAVs, to evaluate patterns of the existing literature. This methodology is widely accepted in the literature and in bibliometrics $[32,33]$.

Specifically, a comprehensive database of gathered data from Scopus [34] was developed. Documents that contain the keywords "UAVs" and "traffic data collection" were selected for the evaluation. The software VOS [35] was applied to examine and analyze the distribution of co-occurrent keywords of the most common keywords outlined below articles' abstracts. The presence of 1104 keywords in 105 publications was confirmed. The threshold of 4 occurrences was adopted. The consistency of the database ensured the validation of the analysis to the context of this study. The final analyzed keywords and their node size are illustrated in Figure 1.

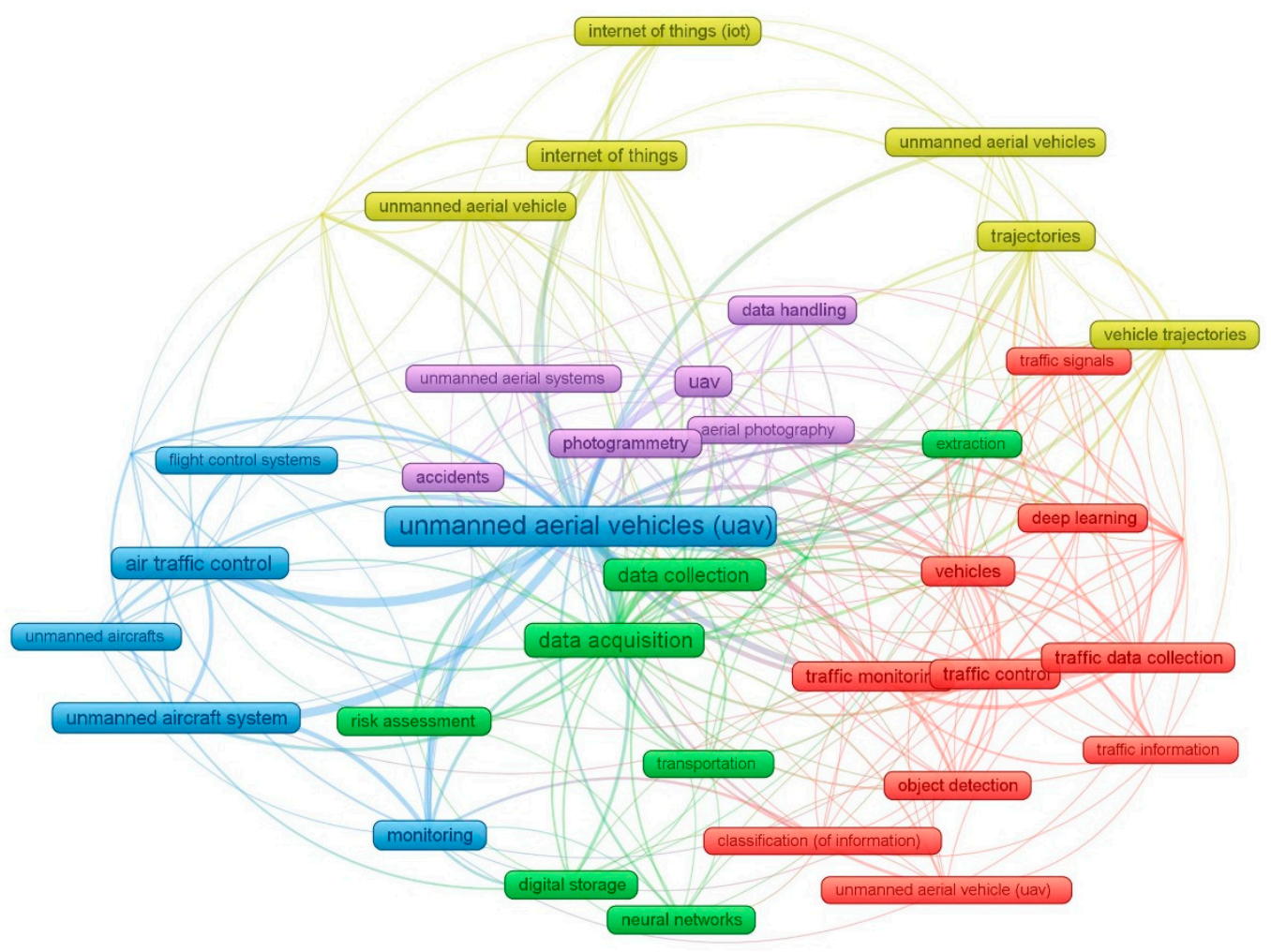

Figure 1. Network visualization of authors' keyword occurrence.

According to the analysis, the higher the keyword and the node, the larger number of articles contain the specific keyword. Moreover, thick lines indicate co-occurrence of the keyword in the literature. Five clusters were developed according to the analysis and each one represents a set of related items. The leading keyword of each cluster and its characteristics in terms of total numbers of occurrences and total link strength of co-occurrences are presented in Table 2. 
Table 2. The top keywords co-occurrence and total link strength.

\begin{tabular}{cccc}
\hline Keyword & Cluster & Occurrences & Total Link Strength \\
\hline unmanned aerial vehicles & blue (1) & 74 & 221 \\
data acquisition & green (2) & 28 & 106 \\
UAV & purple (3) & 12 & 47 \\
traffic data collection & red (4) & 11 & 47 \\
trajectories & yellow (5) & 8 & 36 \\
\hline
\end{tabular}

According to the identified keywords, it can be concluded that various methodologies regarding the extraction of the traffic information exist. The vehicles' detection and classification to extract trajectories for operational and safety analysis, is the main issue the existing literature is dealing with.

Findings and methods of the existing literature were considered to establish a comprehensive framework regarding UAV survey execution and microscopic traffic data acquirement, following the discrete steps of Figure 2.

\section{UAV Traffic Survey}

(A) Preparation of the survey

- Ensure optimal flight planning

- Extract coordinates of GCPs

\section{(B) Survey execution}

- High video resolution

- Low altitudes of the UAV

- Recordings of a nadir point of view

\section{UAV Video processing}

(C) Preliminary process
- Remove of no significant frames
- Identification of specific events
(D) Gens distortion correction
pixels into real-world coordinates
(E) Vehicle trajectory acquisition
(F) Management of vehicle trajectories

Figure 2. The discrete steps to acquire traffic data using UAVs.

The proposed methodology of this paper is structured in such a way to be simply and effectively adopted by researchers and engineers. Detailed naturalistic vehicle trajectories data through UAVs can be extracted following a low-cost method, requiring less demand for high skills or expertise in image processing techniques.

\subsection{UAV Traffic Survey}

\subsubsection{Preparation of the Survey}

UAV flight planning for the collection of the required data depends on several aspects that are critical for ensuring a successful UAV flight operation. According to [26], safety issues (such as prohibited fly zones or need for the existence of sufficient space regarding safety distance from sensitive installments) and climate characteristics (such as the weather conditions) can directly affect the survey execution. Thus, an in-depth flight planning before the execution of the UAV survey is essential.

After the identification of the study area, an appropriate number of ground control points (GCPs) need to be distributed homogenously within the area of interest (Figure 3). 


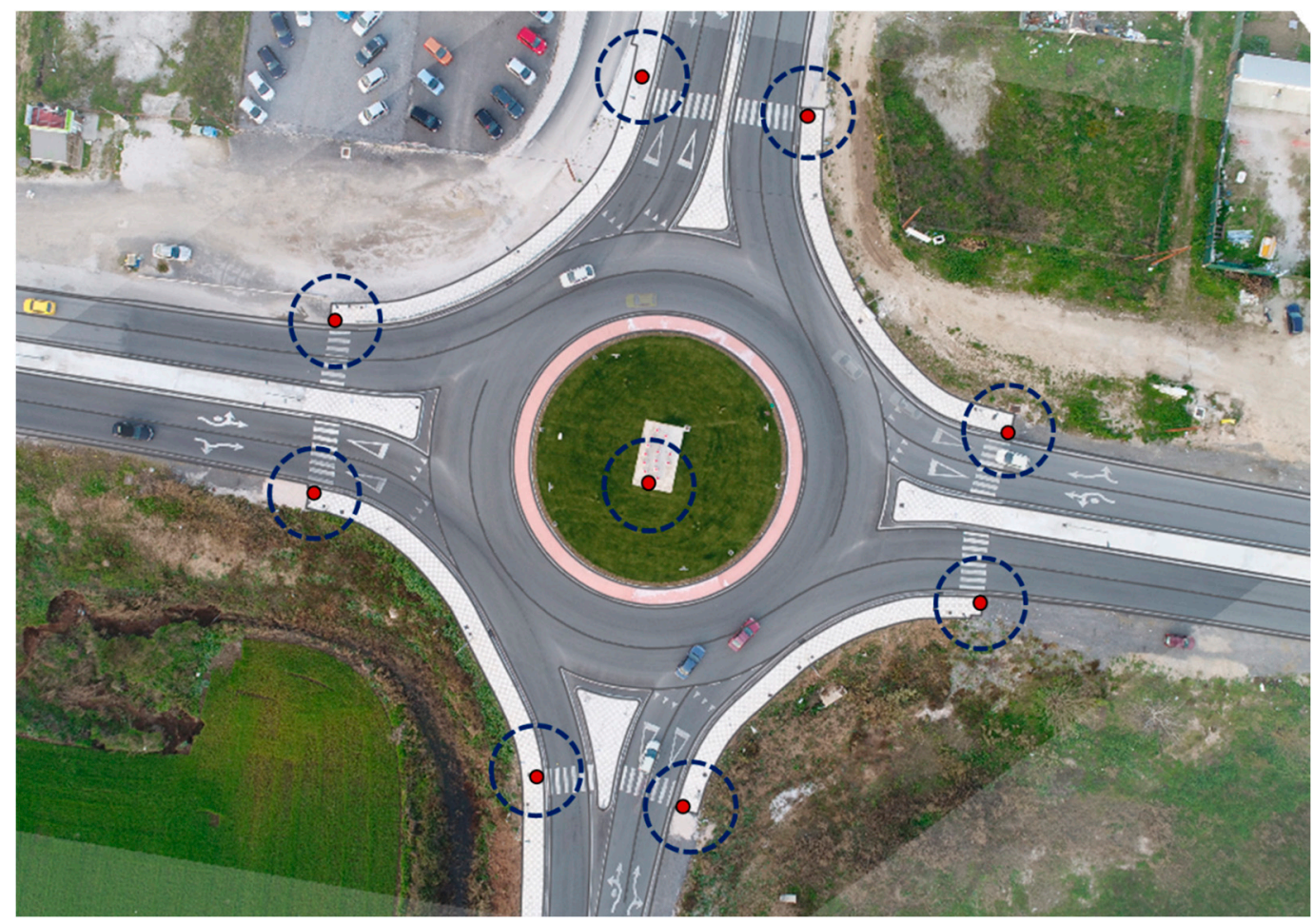

Figure 3. The location of GCPs as distributed homogeneously for the survey.

The aim of this task intends to transform the acquired frames on real-world coordinates through the process of georeferencing. GCPs of well-known coordinates significantly increase the absolute accuracy of the analysis as presented in the following steps. In the case of manual or semi-automatic georeferencing process as in the experiment of this study, GCPs are required to be visible in all acquired frames. Their use intends to correct errors due to UAV tilt (wind forces affect) and camera lens distortion issues. It is noted that the use of natural GCPs (such as corners of manholes and tactile pavements, intersections of white pavement markings, etc.) provides the flexibility to conduct surveys over different time periods, skipping time-consuming field measurements of GCPs coordinates per survey. The restraint of UAVs low battery and the requirement for visible GCPs per each survey means that permanent natural ground control points are an efficient choice. A proper tool can provide the coordinates of the selected ground control points in high accuracy. Figure 4 presents an example of natural GCPs, the coordinates of which are measured using a RTK GNSS receiver.
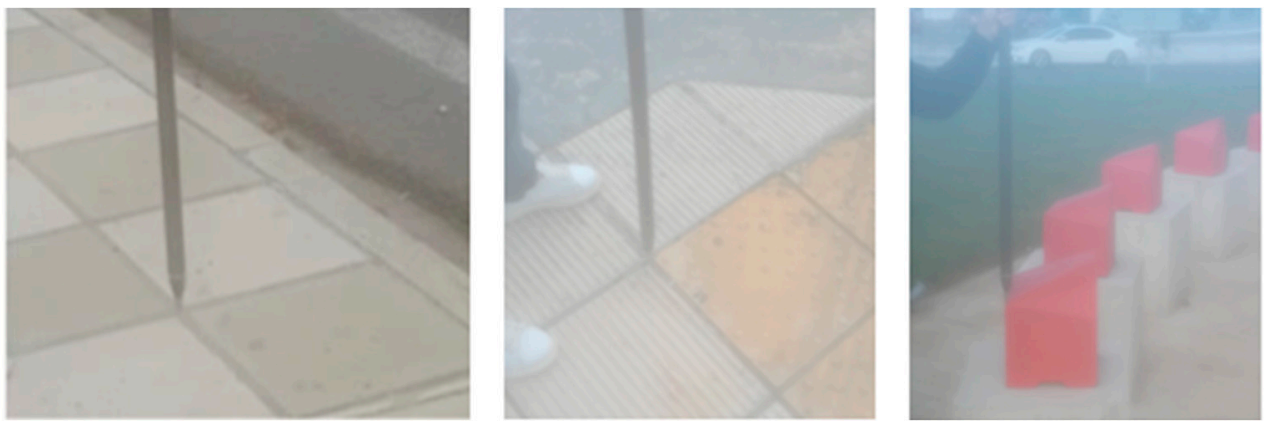

Figure 4. Natural ground control points. 


\subsubsection{Survey Execution}

During the UAV survey execution, the safety and legal issues should be addressed. UAV flight can be handled manually via a controller or automatically according to a predefined route. It is mentioned that both the surveyor and the equipment should not be noticed by road users. Any distraction of drivers' attitude affects the naturalness of the collected data.

There are three main aspects that affect the level of detail of the study and the surveyor should consider during a UAV flight: (a) the video resolution, (b) the altitude of the UAV and (c) the viewing angle. More specifically, it is recommended video recordings be saved at the highest possible quality. High-resolution videos and low flight altitudes optimize the required time regarding the video processing and increase the accuracy and the quality of the study. Videos recorded from an angle require a process of pixel transformation to achieve an orthographic view. This is usually carried out using perspective filters. Thus, recordings of a nadir point of view minimize camera errors and are preferable.

Through these main aspects, the ground sample distance (GSD) can be reduced, enhancing the final accuracy of the analysis. With lower GSD, the identification of the appropriate point regarding vehicle tracking analysis, will be much easier. It is noted that the measured pixel size determines the minimum threshold of the final accuracy.

Finally, the location of the UAV should be stable to minimize the bias in the stabilizing process. A gimbal system attached to the camera can stabilize the recorded shots in an efficient way.

\subsection{UAV Video Processing}

\subsubsection{Preliminary Process}

A preliminary process is required to simplify the video processing. This step includes the following operations: Firstly, no significant frames form videos (take-off and landing) are removed. A stabilization process is required to minimize rough bias. Proper filters contribute to smoother videos and the elimination of camera shakiness. In the following step, specific events according to the scope of the analysis are identified (for example, in a speed analysis the time periods of vehicles on free-flow speed conditions are identified). The video frames of the selected time periods are extracted per second. Finally, the lens distortion of the acquired images needs to be corrected. There are several techniques that can be adopted. A common practice is to transform images by adopting the distortion profile of the implemented camera.

\subsubsection{Geo-Registration}

The transformation of UAV-acquired image pixels into real-world coordinates allows the extraction of vehicle trajectory data into real-world coordinates. The use of a cartesian coordinate system calibrated to a specific scale is a common practice regarding the georegistration process. However, the use of GCPs in the experiments, except for the high accuracy of the analysis and the correction of image distortion, provides a comprehensive dataset that can be managed by several applications, depending on the purpose of the study.

A reference image of the study area is georeferenced according to the known coordinates of the GCPs. The extracted frames of the identified events are then co-registered to the reference image. This process can be carried out either in a manual way, which provides accurate but time-consuming results, or in an automatic way by using pixel matching algorithms, which promises quicker and less accurate results.

\subsubsection{Vehicle Trajectory Acquisition}

To acquire microscopic traffic data, the extraction of accurate vehicle trajectories is essential. Several studies carried out recently are dealing with this issue $[11,20,26,36-39]$. The adopted methodologies can be divided into three main categories: (a) the manual process, (b) the semi-automatic process, and (c) the automatic process technique. The first two methods are more accurate and offer many flexibility advantages, however, they 
are time-consuming. On the other hand, the third method promises quicker results by using detection techniques and tracking algorithms with minimum manpower involved. Nevertheless, this requires high expertise and knowledge of computer vision techniques, while its accuracy on high spatial data such as vehicle trajectories sometimes requires manual effort for the correction of missed trajectories.

\subsubsection{Management of Vehicle Trajectories}

Utilizing the vehicle trajectory data, several applications can be carried out and information of great importance can be provided. Thus, it is required that the vehicle location data be organized in detail on a comprehensive dataset along with supplementary information (such as the type of the vehicle). Researchers and engineers can interpret the measured data to understand the way the vehicles move.

\section{Case Study Experiment}

The described methodology was applied on a multilane roundabout layout where the complexity of driving behavior is challenging as the road geometry provides high uncertainty in driving maneuvers. The method was tested for two different lighting conditions: daylight and night. An analysis on vehicle trajectories was carried out to extract microscopic traffic data such as vehicles speeds, travel time measurements, and the spatial distribution of vehicle maneuvers. The selected multilane roundabouts (Figure 5) are in the cities of (a) Larissa and (b) Thessaloniki (Greece).

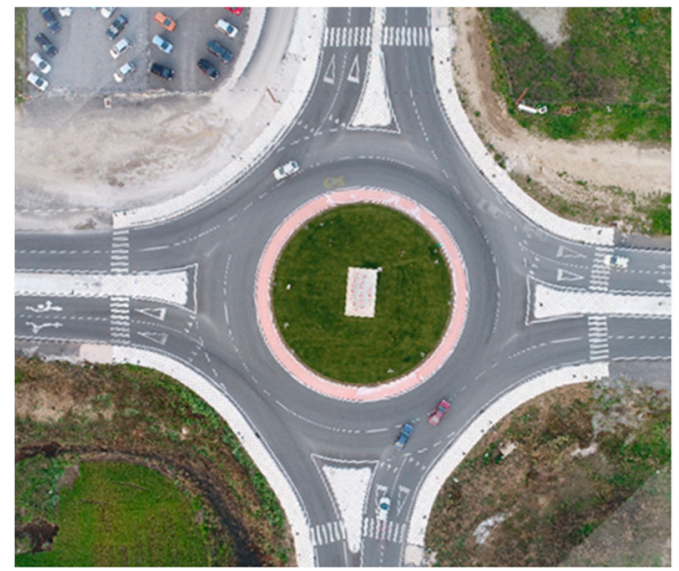

(a)

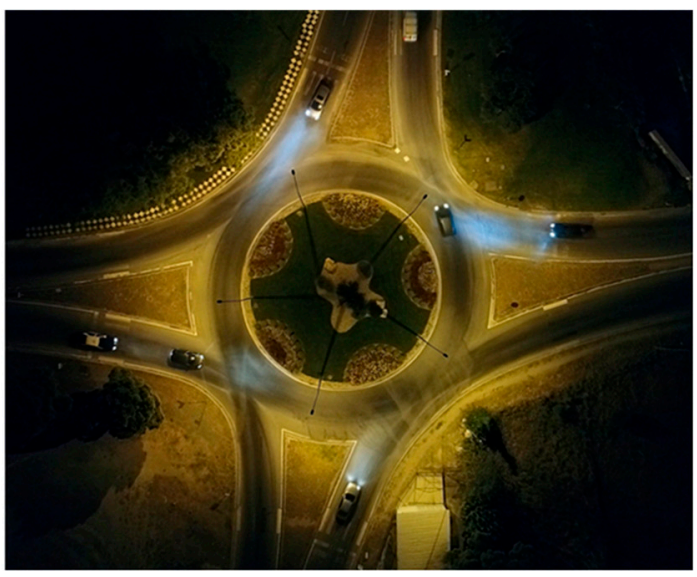

(b)

Figure 5. UAV shot of the examined case studies: (a) Larissa and (b) Thessaloniki.

\subsection{Survey Material and Equipment}

Two types of equipment were used to perform the experiment described in this paper to acquire reliable naturalistic vehicle trajectories data of the roundabouts: (a) a quadcopter UAV (Figure 6) and (b) a RTK GNSS receiver. The selected UAV (DJI Phantom 4 Advanced) can capture videos up to $\mathrm{C} 4 \mathrm{~K}$ analysis with a frame rate of $60 \mathrm{fps}$ and high-resolution images $(5472 \times 3078)$. It is a low-cost UAV and requires less experience to be controlled. Its camera is attached to a gimbal, which stabilize shots. Moreover, its weight (1368 g) and size contribute to better maneuverability, while low altitudes flights achieve high spatial resolution. The RTK GNSS receiver that was used provides reliable and high-accuracy data collection. The position of selected GCPs can be determined in centimeter-level accuracy in real world conditions. Specifically, the accuracy of this equipment examined for a survey is $8 \mathrm{~mm}+1$ ppm (horizontal) and $15 \mathrm{~mm}+1$ ppm (vertical). 


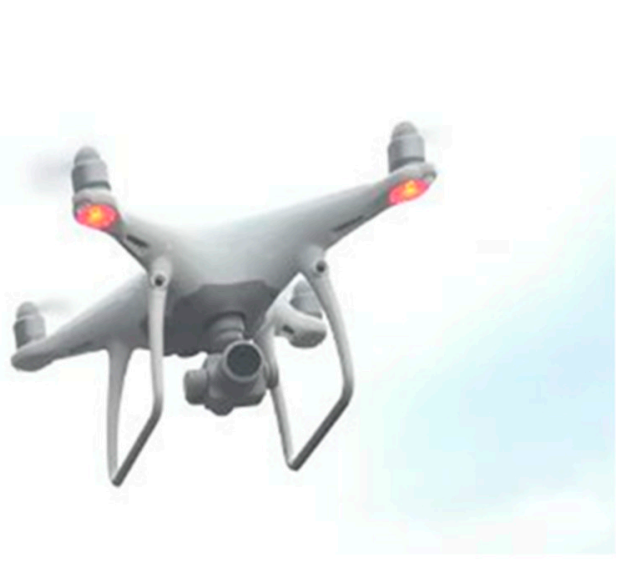

(a)

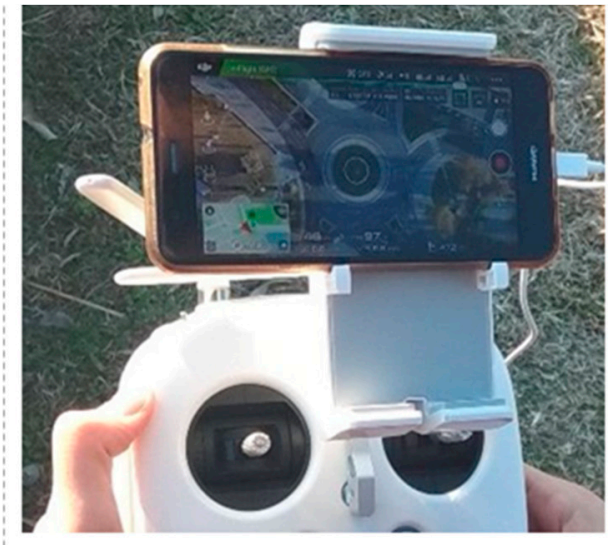

(b)

Figure 6. (a) The quadcopter UAV and (b) the captured study area as presented on the controller during the survey.

\subsection{UAV Survey}

Roundabouts' performance was recorded with the use of the Unmanned Aerial Vehicle (UAV) during summer and autumn of 2019. Data regarding the kinematic characteristics of the vehicles were collected in this experiment.

Field measurements were selected to be conducted during of-peak periods to ensure free flow speed conditions. Real vehicle speeds in unobstructed traffic conditions were collected. Weather conditions were stable and did not affect the vehicle movements. Low flight altitudes of a nadir point of view and high-resolution recordings allowed to measure accurate and naturalistic spatiotemporal phenomena of high detail.

\subsection{Data Processing}

Vehicle trajectories were extracted from the UAV videos following specific steps. These steps aimed to: (a) reduce any bias and increase the accuracy and (b) digitize and study vehicle motion paths.

Firstly, no significant frames from videos (take-off and landing) were removed. A stabilization procedure was followed [40]. Proper filters made videos smoother while camera shakiness was eliminated. Specific events were identified (vehicle through movements on free flow speed conditions) and video frames were extracted for each examined video. Lens distortion of each frame was corrected [41]. The acquired images were georeferenced in high accuracy using an open-source GIS software [42]. The selected geographic reference was the "GGRS87/Greek Grid". The ground sample distance was measured to the value of $35.6 \mathrm{~mm}$ and the maximum value of the RMSE between the real location and the final georeferenced location per each frame was 0.6.

Finally, a digitization process was carried out to acquire vehicle trajectories. Specifically, the center of the front bumper of each vehicle was identified and the coordinates were extracted. Mathematical interpolation based on the known coordinates was used in CAD software using spline curves so the discrete data could be transformed into continuous functions to further study vehicle maneuvers and microscopic traffic data.

\subsection{Experiment Results}

Several applications regarding microscopic traffic data analysis can be carried out regarding the extracted dataset. Understanding the way road users move in roundabouts is of great importance, since many conflicts occur in this type of intersections [43,44].

Figure 7 presents a heatmap of the spatial distribution of vehicle maneuvers according to the extracted vehicle trajectories of the examined case study roundabout. Various driving behavior patterns for through movements on the multilane roundabout are indicated. Improper and unexpected paths are created and that can probably cause unexpected behavior, and therefore potential danger. 


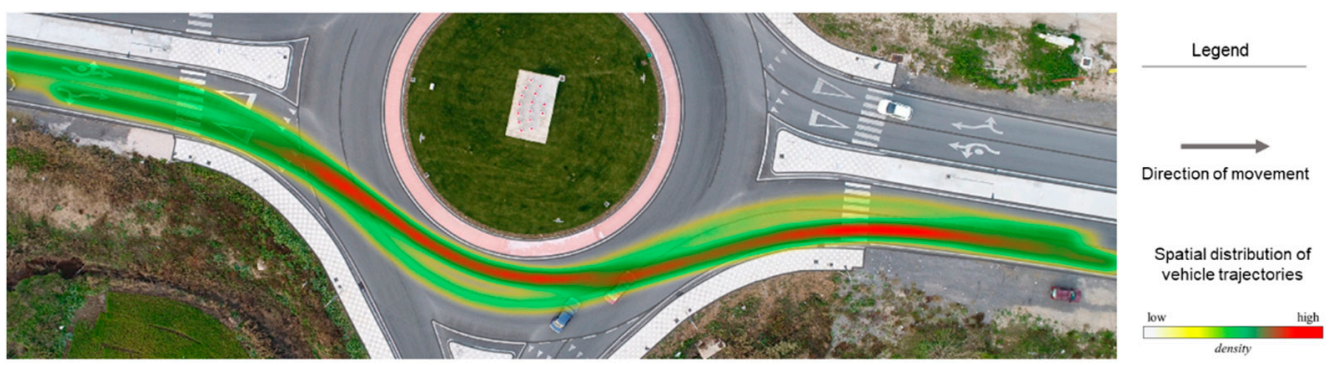

Figure 7. Heatmap of the spatial distribution of vehicle maneuvers of Roundabout A.

To calculate vehicle speeds at the entrance of the roundabout, firstly the proper events were identified. Then, the captured distances per $0.2 \mathrm{~s}$ were calculated and divided by the corresponding time. Considering the measured value of the ground sample distance $(35.6 \mathrm{~mm}$ ) and the RMS error of 0.6 , it is concluded that the maximum error in speed estimation is less than $1 \mathrm{~km} / \mathrm{h}$. An indicative calculated speed profile of a vehicle traversing the roundabout is presented in Figure 8.

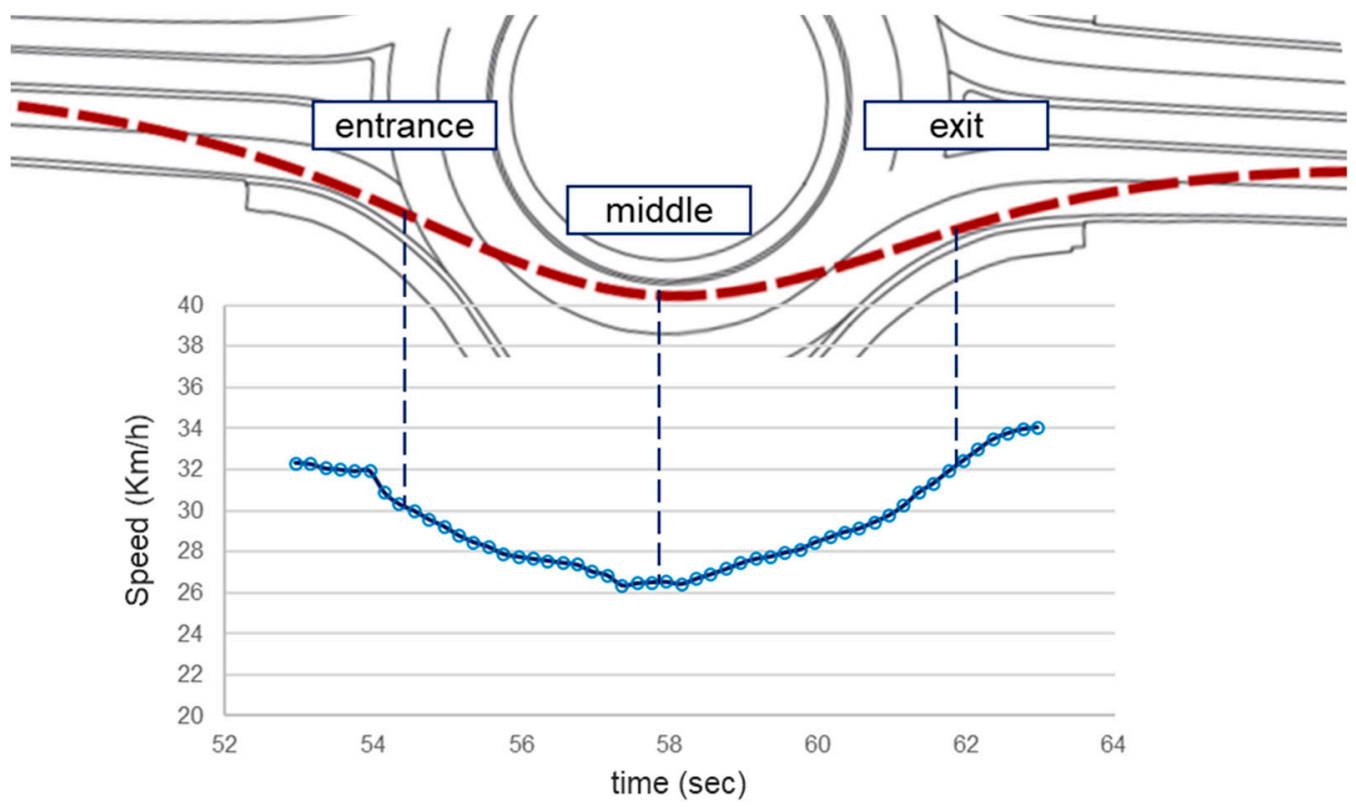

Figure 8. A typical vehicle speed profile has been extracted by processing UAV shots.

The vehicle speed profile indicates significant changes in speeds because of the complexity of the road geometry. The driver reduces the vehicle speed along the entry and the center of the roundabout and then accelerates to the exit lane.

With the second case study roundabout, an analysis regarding the travel time of 31 vehicles passing through the roundabout was conducted. An algorithm was developed and applied to the extracted trajectories. Two detection lines were coded at the entrance and the exit of the roundabout to identify the timestamps of each vehicle passing these sections (Figure 9). Results provide information regarding the travel time of vehicles through-movements. The average travel time of through movements under free-flow speed conditions is $5.14 \mathrm{~s}$. 


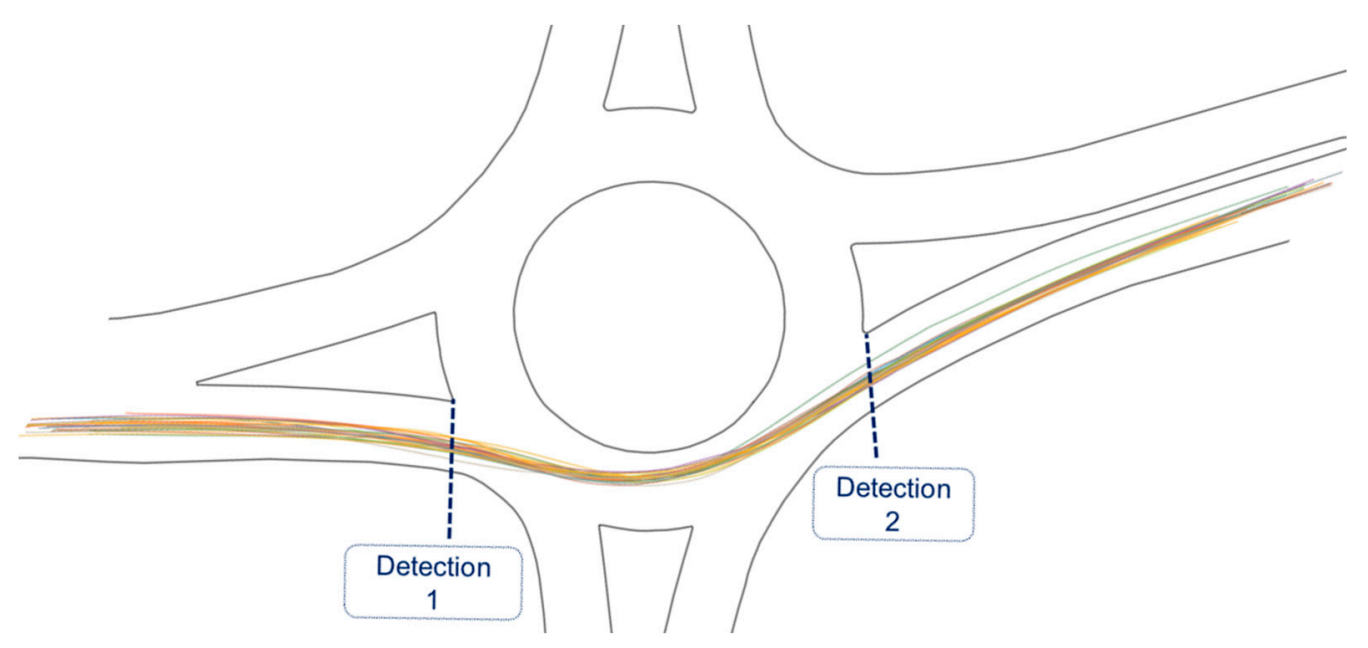

Figure 9. Graphical representation of the vehicle trajectories and the location of coded detectors.

\section{Conclusions}

Research into collecting and measuring reliable, accurate, and naturalistic microscopic traffic data is a fundamental aspect in road network planning scientific literature. According to the literature review and the bibliometric and visualization analysis, it can be concluded that UAVs are recently being used in the transportation field to monitor and analyze the traffic flow. The vehicles' detection and classification to extract trajectories for operational and safety analysis is the main issue the existing literature is dealing with.

The proposed methodology of this paper is structured in such a way to be simply and effectively adopted by researchers and engineers. Detailed and naturalistic vehicle trajectories data can be extracted through UAVs in a low-cost way, requiring less demand for high skills or expertise in image processing techniques.

Two experiments were carried out on different light conditions following the presented framework. Results indicate that the accuracy of the extracted microscopic traffic data is high enough as the maximum error in speed estimation is less than $1 \mathrm{~km} / \mathrm{h}$. The measured data can be interpreted and applied to several studies. In these experiments, vehicle trajectories were analyzed to extract vehicles speeds, travel time measurements, and the spatial distribution of vehicle maneuvers. The extracted dataset can be utilized and be applied on various traffic studies (gap-acceptance analysis, road safety analysis, study on lane-change behavior, calibration of car-following models, etc.).

The limitations of the study are related mainly to the technical issues of the UAV technology and the climate characteristics of the examined area. More specifically, the restraint of the low battery duration of UAVs and the weather conditions of the study area (rain) resulted in short video recordings and time-consuming field experiments.

\section{Discussion and Final Remarks}

Accurate and high detailed naturalistic microscopic traffic data are essential for reliable traffic analysis and efficient calibration process of traffic models. The forthcoming applications of intelligent transport systems on vehicles and infrastructure require sufficient tools to calibrate existing models on more complex situations.

UAVs are one of the most emerging technologies being used recently in transportation field to monitor and analyze the traffic flow. The increased commercial market share of this technology during the last years and the developed traffic applications entail a promising contribution to the transportation sector.

Several applications and methodologies regarding the acquirement of microscopic traffic data have been tested until now. This paper aims to add value to the existing literature. A comprehensive framework for extracting naturalistic vehicle trajectories recorded by UAVs is proposed. The presented methodology describes a highly accurate 
and low-cost method to conduct UAV traffic surveys and extract the required information, considering the methods and the limitations reported in previous studies. Researchers and engineers can efficiently apply the structured process as it is simplified and does not require great skills or expertise in automated image processing techniques.

Overall, the benefits of using UAVs on microscopic traffic data collection surveys, as explained in the literature review and highlighted in the presented experiment are great of importance. However, there are still many limitations and issues that need to overcome during the next years to optimize the general process of UAV traffic surveys. Thus, the ability to select and implement UAVs as an efficient traffic survey tool depends on many factors.

Further research is required regarding the acceptability of UAVs on traffic data collection surveys. Specifically, a comprehensive guiding framework under what circumstances this technology should be preferred or discouraged compared to other traditional methods will enhance the overall process of the examined traffic analysis. Finally, due to the forthcoming applications of intelligent transport systems on vehicles and infrastructure, experiments on the capabilities of UAVs regarding real-time traffic data collection techniques and methods of sharing the traffic information to road users are expected to enhance UAVs technology in the transportation engineering sector.

Author Contributions: A.A. and F.K., conceptualization; A.A., methodology; A.A., writing; F.K. review, supervision. All authors have read and agreed to the published version of the manuscript.

Funding: This research received no external funding.

Informed Consent Statement: Not applicable.

Conflicts of Interest: The authors declare no conflict of interest.

\section{References}

1. Shen, J.; Yang, G. Crash Risk Assessment for Heterogeneity Traffic and Different Vehicle-Following Patterns Using Microscopic Traffic Flow Data. Sustainability 2020, 12, 9888. [CrossRef]

2. Michael, P.G.; Leeming, F.C.; Dwyer, W.O. Headway on urban streets: Observational data and an intervention to decrease tailgating. Transp. Res. Part F Traffic Psych. Behav. 2000, 3, 55-64. [CrossRef]

3. Durrani, U.; Lee, C.; Maoh, H. Calibrating the Wiedemann's vehicle-following model using mixed vehicle-pair interactions. Transp. Res. Part C Emerg. Technol. 2016, 67, 227-242. [CrossRef]

4. Zafri, N.M.; Rony, A.I.; Adri, N. Analysis of Pedestrian Crossing Speed and Waiting Time at Intersections in Dhaka. Infrastructures 2019, 4, 39. [CrossRef]

5. Anagnostopoulos, A.; Kehagia, F. CAVs and Roundabouts: Research on traffic impacts and design elements. Transp. Res. Proc. 2020, 49, 83-94. [CrossRef]

6. García Cuenca, L.; Guindel, C.; Aliane, N.; Armingol, J.M.; Fernández Andrés, J. Dataset Construction from Naturalistic Driving in Roundabouts. Sensors 2020, 20, 7151. [CrossRef] [PubMed]

7. Hoogendoorn, S.; Knoop, V. Traffic flow theory and modelling. In The Transport System and Transport Policy: An Introduction; van Wee, B., Annema, J.A., Banister, D., Eds.; Edward Elgar Publishing Limited: Cheltenham, UK, 2012; pp. $125-159$.

8. Coifman, B.; Wu, M.; Redmill, K.; Thornton, D. Collecting ambient vehicle trajectories from an instrumented probe vehicle: High quality data for microscopic traffic flow studies. Transp. Res. Part C Emerg. Technol. 2016, 72, 254-271. [CrossRef]

9. Li, L.; Jiang, R.; He, Z.; Chen, X.; Zhou, Z. Trajectory data-based traffic flow studies: A revisit. Emerg. Technol. 2020, 114, 225-240. [CrossRef]

10. Dingus, T.A.; Klauer, S.G.; Neale, V.L.; Petersen, A.; Lee, S.E.; Sudweeks, J.; Perez, M.A.; Hankey, J.; Ramsey, D.; Gupta, S.; et al. The 100-Car Naturalistic Driving Study, Phase II-Results of the 100-Car Field Experiment; Technical Report No. DOT HS 810 593; Virginia Tech Transportation Institute: Blacksburg, Virginia, 2006.

11. Anagnostopoulos, A.; Kehagia, F. Evaluating Fastest Path Procedures on Roundabouts by Extracting Vehicle Trajectories from Unmanned Aerial Vehicles. In Advances in Intelligent Systems and Computing: Advances in Mobility-as-a-Service System. CSUM 2020. Advances in Intelligent Systems and Computing; Nathanail, E.G., Adamos, G., Karakikes, I., Eds.; Springer: Cham, Switzerland, 2020; Volume 1278, pp. 1001-1011. [CrossRef]

12. Hidas, P.; Wagner, P. Review of Data Collection Methods for Microscopic Traffic Simulation. In Proceedings of the World Conference on Transport Research, Istanbul, Turkey, 8 June 2004.

13. Punzo, V.; Simonelli, F. Analysis and Comparison of Microscopic Traffic Flow Models with Real Traffic Microscopic Data. Transp. Res. Rec. J. Transp. Res. Board 2005, 1934, 53-63. [CrossRef] 
14. Giuseppe, G.; Alessandro, V.; Fedel, F.S.; Carmine, D.F.; Vittorio, A.; Daniele, R.; Vincenzo, G. Using Smartphones as a Toll to Capture Road Traffic Attributes. Appl. Mech. Mater. 2013, 432, 513-519. [CrossRef]

15. Kim, E.J.; Park, H.C.; Ham, S.W.; Kho, S.Y.; Kim, D.K. Extracting Vehicle Trajectories Using Unmanned Aerial Vehicles in Congested Traffic Conditions. J. Adv. Transp. 2019, 2019. [CrossRef]

16. Punzo, V.; Borzacchiello, M.T.; Ciuffo, B. On the assessment of vehicle trajectory data accuracy and application to the Next Generation SIMulation (NGSIM) program data. Transp. Res. Part C Emerg. Technol. 2011, 19, 1243-1262. [CrossRef]

17. Iqbal, M.S.; Choundhury, F.C.; Wang, P.; Gonzalez, C.M. Development of origin-destination matrices using mobile phone call data. Transp. Res. Part C Emerg. Technol. 2014, 40, 63-74. [CrossRef]

18. Zhu, M.; Wang, X.; Tarko, A.; Fang, S. Modeling car-following behavior on urban expressways in Shanghai: A naturalistic driving study. Transp. Res. Part C Emerg. Technol. 2018, 93, 425-445. [CrossRef]

19. Shrestha, D.; Lovell, J.D.; Tripodis, Y. Hardware and software for collecting microscopic trajectory data on naturalistic driving behavior. J. Intell. Transp. Syst. 2017, 21, 202-213. [CrossRef]

20. Salvo, G.; Caruso, L.; Scordo, A.; Guido, G.; Vitale, A. Traffic data acquirement by unmanned aerial vehicle. Eur. J. Remote Sens. 2017, 50, 343-351. [CrossRef]

21. Bicici, S.; Zeybek, M. An approach for the automated extraction of road surface distress from a UAV-derived point cloud. Autom. Constr. 2021, 122, 103475. [CrossRef]

22. Outay, F.; Mengash, H.A.; Adnan, M. Applications of unmanned aerial vehicle (UAV) in road safety, traffic and highway infrastructure management: Recent advances and challenges. Transp. Res. Part A Policy Pract. 2020, 141, 116-129. [CrossRef]

23. Cardenal, J.; Fernández, T.; Pérez-García, J.L.; Gómez-López, J.M. Measurement of Road Surface Deformation Using Images Captured from UAVs. Remote Sens. 2019, 11, 1507. [CrossRef]

24. Almeshal, A.M.; Alenezi, M.R.; Alshatti, A.K. Accuracy Assessment of Small Unmanned Aerial Vehicle for Traffic Accident Photogrammetry in the Extreme Operating Conditions of Kuwait. Information 2020, 11, 442. [CrossRef]

25. Trubia, S.; Curto, S.; Severino, A.; Arena, F.; Puleo, L. The use of UAVs for civil engineering infrastructures. AIP Conf. Proc. 2021, $2343,110012$.

26. Khan, M.; Ectors, W.T.; Bellemans, D.; Jassens, D.; Wets, G. UAV-Based Traffic Analysis: A Universal Guiding Framework Based on Literature Survey. Transp. Res. Proc. 2017, 22, 541-550. [CrossRef]

27. Guido, G.; Gallelli, V.; Rogano, D.; Vitale, A. Evaluating the accuracy of the vehicle tracking data obtained from Unmanned Aerial Vehicles. Int. J. Transp. Sci. Tech. 2016, 5, 136-151. [CrossRef]

28. Kanistras, K.; Martins, G.; Rutherford, M.J.; Valavanis, K.P. Survey of Unmanned Aerial Vehicles (UAVs) for Traffic Monitoring. In Handbook of Unmanned Aerial Vehicles; Valavanis, K., Vachtsevanos, G., Eds.; Springer: Dordrecht, The Netherlands, 2014; pp. 2643-2666. [CrossRef]

29. Chen, P.; Zeng, W.; Yu, G.; Wang, Y. Surrogate Safety Analysis of Pedestrian-Vehicle Conflict at Intersections Using Unmanned Aerial Vehicle Videos. J. Adv. Transp. 2017, 2017. [CrossRef]

30. Barmpounakis, N.E.; Vlahogianni, E.; Golias, C.J.; Babinec, A. How accurate are small drones for measuring microscopic traffic parameters? Int. J. Transp. Res. 2019, 11, 332-340. [CrossRef]

31. Barmpounakis, N.E.; Vlahogianni, E.; Golias, J.C. Extracting Kinematic Characteristics from Unmanned Aerial Vehicles. In Proceedings of the Transportation Research Board 95th Annual Meeting, Washington, DC, USA, 10-14 January 2016.

32. Badassa, B.B.; Sun, B.; Qiao, L. Sustainable Transport Infrastructure and Economic Returns: A Bibliometric and Visualization Analysis. Sustainability 2020, 12, 2033. [CrossRef]

33. Guo, Y.-M.; Huang, Z.-L.; Guo, J.; Li, H.; Guo, X.-R.; Nkeli, M.J. Bibliometric Analysis on Smart Cities Research. Sustainability 2019, 11, 3606. [CrossRef]

34. Scopus. Available online: https:/ / www.scopus.com/ (accessed on 17 March 2021).

35. VOSviewer. Available online: https://www.vosviewer.com/ (accessed on 17 March 2021).

36. Meng, L.; Wang Xiao, H.; Shi, K. Traffic Conflict Identification Technology of Vehicle Intersection Based on Vehicle Video Trajectory Extraction. Procedia Comput. Sci. 2017, 109, 963-968. [CrossRef]

37. Khan, M.A.; Ectors, W.; Bellemans, T.; Janssens, D.; Wets, G. Unmanned Aerial Vehicle-Based Traffic Analysis: A Case Study for Shockwave Identification and Flow Parameters Estimation at Signalized Intersections. Remote Sens. 2018, 10, 458. [CrossRef]

38. Salvo, G.; Caruso, L.; Scordo, A. Urban traffic analysis through an UAV. Procedia Soc. Behav. Sci. 2014, 111, 1083-1091. [CrossRef]

39. Krajewski, R.; Bock, J.; Kloeker, L.; Eckstein, L. The highD Dataset: A Drone Dataset of Naturalistic Vehicle Trajectories on German Highways for Validation of Highly Automated Driving Systems. In Proceedings of the 21st International Conference on Intelligent Transportation Systems (ITSC), Maui, HI, USA, 4-7 November 2018.

40. Deshaker. Available online: http:/ / www.guthspot.se/video/deshaker.htm (accessed on 17 March 2021).

41. Adobe Lightroom. Available online: https://lightroom.adobe.com (accessed on 17 March 2021).

42. QGIS Geographic Information System. Available online: http:/ / qgis.org (accessed on 17 March 2021).

43. Anagnostopoulos, A.; Kehagia, F. Turbo-roundabouts as an alternative to roundabouts in terms of traffic safety, capacity and pollutant emissions. In Proceedings of the 7th Pan-Hellenic Road Safety Conference, Larissa, Greece, 11-12 October 2018.

44. Damaskou, E.; Kehagia, F.; Pitsiava-Latinopoulou, M.; Anagnostopoulos, A. Factors Influencing Quality of Service in Road Safety at Urban Roundabouts, Based on Driver's Perception. In Proceedings of the 48th European Transport Conference, Milan, Italy, 9-11 September 2020. 